Proceedings the 44th International School and Conference on the Physics of Semiconductors "Jaszowiec 2015", Wisła 2015

\title{
Influence of Geometrical Parameters on the Transport Characteristics of Gated Core-Multishell Nanowires
}

\author{
T. Palutkiewicz, M. Woøoszyn, P. Wójcik, J. Adamowski and B.J. Spisak* \\ AGH University of Science and Technology, Faculty of Physics and Applied Computer Science, \\ al. Mickiewicza 30, 30-059 Kraków, Poland
}

\begin{abstract}
Calculations of the current-voltage characteristics of the core-multishell nanowires for different radii of the core and various thicknesses of the shells are presented. A role of the conducting core and shells in the coherent transport under the influence of the gate voltage is discussed.
\end{abstract}

DOI: 10.12693/APhysPolA.129.A-111

PACS: 72.80.Ey, 73.63.Nm, 85.35.-p

\section{Introduction}

Availability of the core-multishell (CMS) nanowires made of the III-V semiconductor materials caused an increasing interest in the electronic properties of such nanostructures [1]. This is partly due to the possible applications in electronics as vertical transistors with the operating characteristics controlled by electrostatic gates [1, 2]. Various papers published in last few years indicate that these types of wires can have electrical performance much better than the planar structures, e.g., smaller resistance [3] and larger transconductance [1, 3].

In this report we investigate the influence of the radius of the core and the thicknesses of the shells on the coherent propagation of the conduction electrons through the CMS nanowires in the presence of the surrounding gate.

\section{Model and calculations}

For the present calculations we consider a single cylindrical semiconductor CMS nanowire, which consists of InGaAs core placed inside InP inner shell enclosed by InGaAs outer shell. It is surrounded by a gate placed asymmetrically in the vicinity of the drain electrode, and separated from the outer shell by the insulator layer made of $\mathrm{Hf}_{0.8} \mathrm{Al}_{0.2} \mathrm{O}$. The nanowire is modelled as a system of ultrasmooth concentric layers with various lengths $\left(l_{i}\right)$ and thicknesses $\left(t_{i}\right)$, as it is shown in Fig. 1.

Calculations are performed on uniform computational grid in the following steps. at first, the electrostatic potential is determined in the whole system under the applied gate voltage $\left(v_{\mathrm{g}}\right)$ as a numerical solution of the three-dimensional (3d) laplace equation

$$
\nabla \cdot[\epsilon(\boldsymbol{r}) \nabla V(\boldsymbol{r})]=0 \text {, }
$$

with the Dirichlet boundary conditions. Then, we use the adiabatic approximation [4] to solve the 3D Schrödinger equation

\footnotetext{
*corresponding author; e-mail: bjs@agh.edu.pl
}

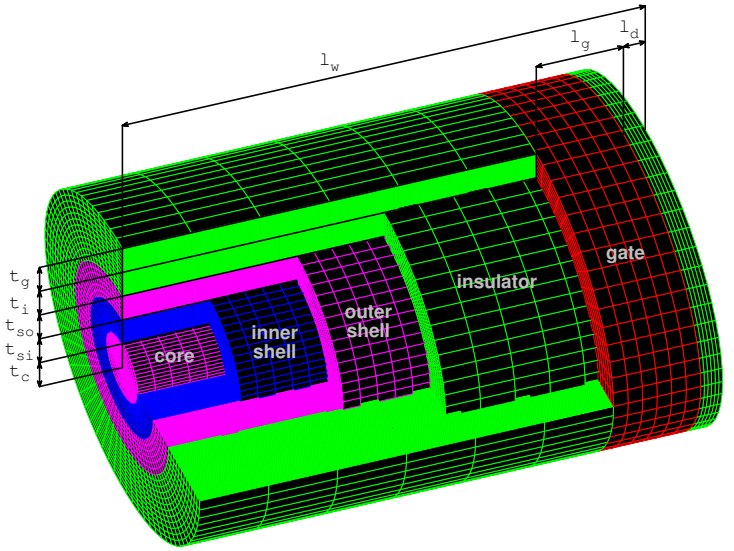

Fig. 1. Model of core-multishell nanowire (not in scale).

$$
\left[-\frac{\hbar^{2}}{2 m_{0}} \nabla \frac{1}{m(\boldsymbol{r})} \nabla+U(\boldsymbol{r})\right] \psi(\boldsymbol{r})=E \psi(\boldsymbol{r}),
$$

where $m(\boldsymbol{r})$ is the position-dependent effective mass, and the total potential energy $U(\boldsymbol{r})$ consists of three terms: the potential energy resulting from the Laplace equation, hard-wall confinement energy, and the effective potential energy associated with the conduction band bottom characterizing each layer of the CMS nanowire. Due to the axial symmetry of the nanosystem the potential energy has a quasi-separable form. This allows us to find the eigenstates for perpendicular $2 \mathrm{D}$ cross-sections along the nanowire, which are used to construct the longitudinal potential energy profiles. The drain-source voltage $V_{\mathrm{ds}}$ is then applied, and the energy-dependent transmission coefficients are calculated using the 1D quantum transmission boundary method (QTBM) [5] which finally allows us to obtain the electronic current according to the formula [6]:

$$
\begin{aligned}
& I_{\mathrm{d}}\left(V_{\mathrm{ds}}, V_{\mathrm{g}}\right)= \\
& \frac{2 e}{h} \sum_{n} \int_{-\infty}^{\infty} \mathrm{d} E \mathcal{T}_{n}\left(E ; V_{\mathrm{ds}}, V_{\mathrm{g}}\right)\left[f_{\mathrm{s}}(E)-f_{\mathrm{d}}(E)\right],
\end{aligned}
$$


where $\mathcal{T}_{n}\left(E ; V_{\mathrm{ds}}, V_{\mathrm{g}}\right)$ is the transmission coefficient, and $f_{\mathrm{s}(\mathrm{d})}(E)=\left[1+\exp \left(\left(E-\mu_{\mathrm{s}(\mathrm{d})}\right) /\left(k_{\mathrm{B}} T\right)\right)\right]^{-1}$ is the Fermi-Dirac distribution function at temperature $T$ with the electrochemical potential $\mu_{\mathrm{s}}$ of the source, and $\mu_{\mathrm{d}}=$ $\mu_{\mathrm{s}}-e V_{\mathrm{ds}}$ of the drain.

\section{Results}

The model of CMS nanowire presented in Sect. 2 has been used to find the transport properties of such nanosystems at $T=4 \mathrm{~K}$ and for $\mu_{\mathrm{s}}=10 \mathrm{meV}$. For this purpose, we calculate the current-voltage $(I-V)$ characteristics, varying the geometric parameters of the CMS nanowire layers in two ways.

In the first case, we symmetrically broaden the insulating layer (the inner shell) which is placed between the conducting layers (the core and the outer shell) of equal thicknesses (cf. insets in Fig. 2b,c). The results presented in Fig. 2a show that increase of the insulating layer thickness reduces the current. Our calculations also indicate that above a certain critical thickness the current is negligible, and that this critical thickness depends neither on the gate voltage (Fig. 2a) nor on the drain-source voltage (Fig. 2b,c).
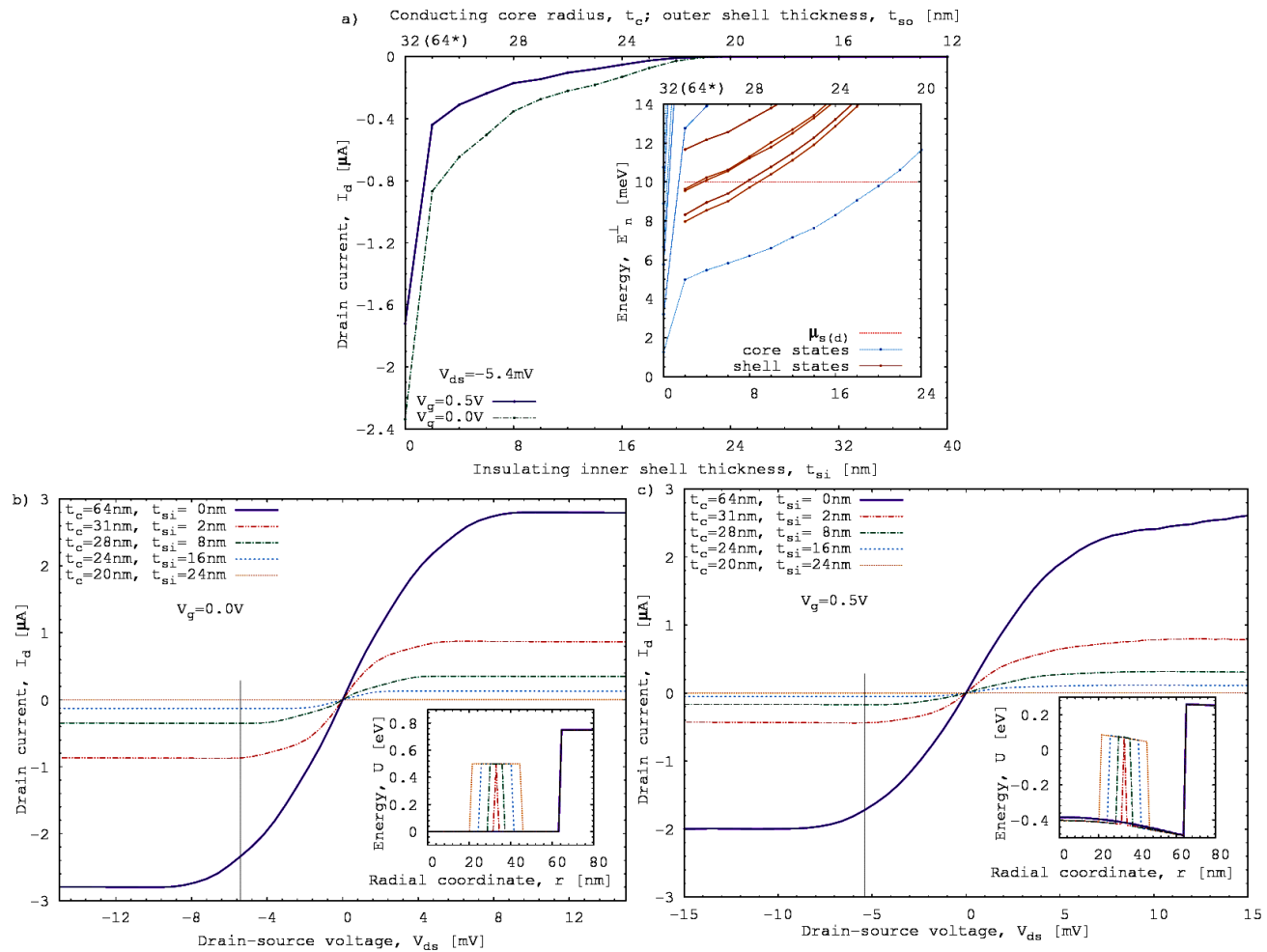

Fig. 2. (a) Comparison of drain current obtained at $V_{\mathrm{ds}}=-5.4 \mathrm{mV}$ under different gate voltages and with increasing thickness $t_{\text {is }}$ of insulating inner shell placed symmetrically around the half of wire's radius. Symbol $*$ indicates that for $t_{\mathrm{is}}=0$ the conducting layers are not separated, and the whole CMS structure reduces to a core with $64 \mathrm{~nm}$-radius. Inset: energy levels at source and drain contacts. (b), (c): Current-voltage characteristics of nanowires with different shell thicknesses at $V_{\mathrm{g}}=0$ (b) and $V_{\mathrm{g}}=0.5 \mathrm{~V}$ (c). Insets: potential energy profiles as a function of $r$ in the middle of the gate.

In the second case, we increase the radius of the conducting core, $t_{c}$, while at the same time we decrease the thickness of the external conducting layer, $t_{\mathrm{os}}$, and keep the thickness of the insulating layer, $t_{\text {is }}$, constant (cf. insets in Fig. 3b,c). Figure 3a shows that increase of $t_{c}$ results in decrease of current, which becomes equal zero for $t_{\mathrm{c}} \leq 20 \mathrm{~nm}$.

Additionally, in both cases the $I-V$ characteristics were calculated at various gate voltages, see Figs. 2b,c and $3 \mathrm{~b}, \mathrm{c}$. In each case the current saturates at a maximum value depending on the thickness of the insulating layer and its distance from the nanowire axis.
The positive gate voltage considerably reduces the current at negative $V_{\mathrm{ds}}$, whereas for positive $V_{\mathrm{ds}}$ the $I-V$ characteristics is only slightly modified.

It should be noted that the decrease of the core radius is accompanied by the increase of the thickness of the external conducting layer, which results in larger total cross-sectional area of conducting layers. In spite of this, the current decreases, which suggests that in the examined range of geometric parameters the core plays the main role in the electronic transport, while the conducting shell has much smaller influence. 


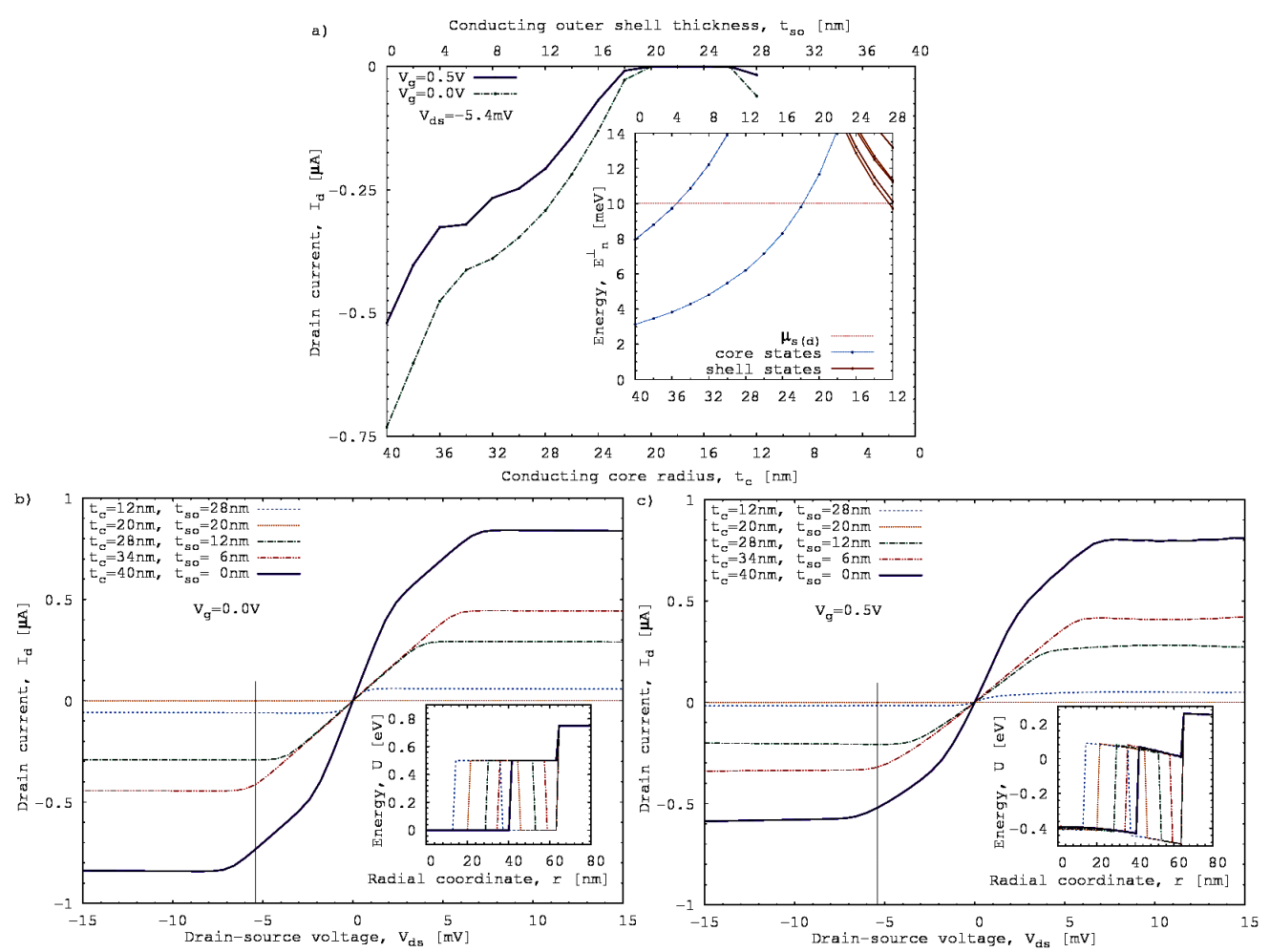

Fig. 3. (a) Comparison of drain current obtained at $V_{\mathrm{ds}}=-5.4 \mathrm{mV}$ under different gate voltages and with increasing radius of conducting core at constant thickness of insulating inner shell. Inset: energy levels at source and drain contacts. (b), (c) Current-voltage characteristics of nanowires with different shell thicknesses at $V_{\mathrm{g}}=0$ (b) and $V_{\mathrm{g}}=0.5 \mathrm{~V}$ (c). Inset: potential energy profiles as a function of $r$ in the middle of the gate.

\section{Discussion}

The results of presented calculations demonstrate that for smaller thickness of any of the conducting layers (i.e., smaller $t_{\mathrm{c}}$ or $t_{\mathrm{os}}$ ), the energies of the transversal states are larger and the gaps between them are wider, as it is shown in Figs. 2a and 3a. The ground-level energy defines the lower limit of the energy range in which the transmission coefficient is not equal zero, while the upper limit is given by the Fermi-Dirac distribution. This means that for the narrow conducting layers the number of open transport channels is small. If the conducting layers are well separated, so that tunnelling between them is infeasible, we can consider them separately. Under such conditions, the narrower layer has a limited impact on the current. For the analysed radii and thicknesses, the contribution of the central core is much larger than that of the external conducting layers.

\section{Conclusions}

The presented results indicate that the core plays the dominant role in the coherent transport of electrons in the analysed core-multishell nanowires. The insulating shells reduce the current as their thicknesses increase or when they are placed closer to the axis of the nanowire.
This is caused by the decreasing number of channels in the transport window and their smaller throughputs which are proportional to the thicknesses of the conducting layers.

\section{Acknowledgments}

This project was supported by the National Science Centre, Poland under grant DEC2011/03/B/ST3/00240.

\section{References}

[1] K. Tomioka, M. Yoshimura, T. Fukui, Nature 488, 189 (2012).

[2] G. Larrieu, X.-L. Han, Nanoscale 5, 2437 (2013).

[3] B. Ganjipour, S. Sepeheri, A.W. Dey, O. Tizno, B.M. Borg, K.A. Dick, L. Samuelson, L.-E. Wernersson, C. Thelander, Nanotechnology 25, 425201 (2014).

[4] M. Wołoszyn, B.J. Spisak, J. Adamowski, P. Wójcik, J. Phys. Condens. Matter 26, 325301 (2014).

[5] C.S. Lent, D.J. Kirkner, J. Appl. Phys. 67, 6353 (1990).

[6] D.K. Ferry, S.M. Goodnick, J. Bird, Transport in Nanostructures, Cambridge University Press, Cambridge 2009 . 